\title{
Transcriptomic changes throughout post-hatch development in Gallus gallus pituitary
}

Elizabeth M Pritchett', Susan J Lamont² and Carl J Schmidt

'Animal and Food Science, University of Delaware, Newark, Delaware, USA

${ }^{2}$ Animal Science, lowa State University, Ames, lowa, USA
Correspondence should be addressed to E M Pritchett

Email

empritch@udel.edu

\begin{abstract}
The pituitary gland is a neuroendocrine organ that works closely with the hypothalamus to affect multiple processes within the body including the stress response, metabolism, growth and immune function. Relative tissue expression ( $\mathrm{rEx}$ ) is a transcriptome analysis method that compares the genes expressed in a particular tissue to the genes expressed in all other tissues with available data. Using rEx, the aim of this study was to identify genes that are uniquely or more abundantly expressed in the pituitary when compared to all other collected chicken tissues. We applied rEx to define genes enriched in the chicken pituitaries at days 21,22 and 42 post-hatch. rEx analysis identified 25 genes shared between all time points, 295 genes shared between days 21 and 22 and 407 genes unique to day 42 . The 25 genes shared by all time points are involved in morphogenesis and general nervous tissue development. The 295 shared genes between days 21 and 22 are involved in neurogenesis and nervous system development and differentiation. The 407 unique day 42 genes are involved in pituitary development, endocrine system development and other hormonally related gene ontology terms. Overall, rEx analysis indicates a focus on nervous system/tissue development at days 21 and 22. By day 42, in addition to nervous tissue development, there is expression of genes involved in the endocrine system, possibly for maturation and preparation for reproduction. This study defines the transcriptome of the chicken pituitary gland and aids in understanding the expressed genes critical to its function and maturation.
\end{abstract}

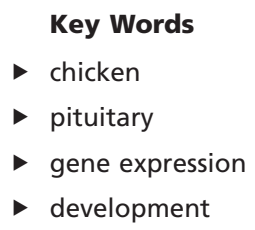

Journal of Molecular Endocrinology (2017) 58, 43-55

\section{Introduction}

The avian pituitary gland is a neuroendocrine organ that works closely with the hypothalamus to regulate multiple processes within the body including the stress response, reproduction, metabolism, growth and immune function. The hypothalamus receives neuronal and endocrine signals from the brain and body and synthesizes releasing hormones that are sent to the pituitary gland to either inhibit or stimulate the production of several hormones that will directly affect other tissues (i.e. growth hormone $(\mathrm{GH})$ ) or stimulate the release of further protein products (i.e. adrenocorticotropic hormone (ACTH)). The posterior pituitary gland, or the neurohypophysis, is composed of nervous tissue and is connected to the hypothalamus via the median eminence. Neurosecretory terminals within the posterior pituitary store and release arginine, vasotocin (AVT) and mesotocin that are synthesized in the supraoptic nucleus and paraventricular nucleus of the hypothalamus, respectively. Releasing hormones synthesized by the hypothalamus are transported to the anterior pituitary gland through the hypophyseal blood 
portal system and regulate the production and release of various endocrine hormones (ACTH, GH, LH, FSH and TSH). Relative tissue expression analysis ( $\mathrm{rEx}$ ) was used to compare the chicken pituitary gland transcriptome to the transcriptome of all other tissues our laboratory has analyzed in the broiler chicken across three time points: days 21,22 and 42 post-hatch. Previous work focusing on the morphometric changes of broiler chickens post-hatch identified two distinct growth periods: one from day of hatch through day 14 , and one from day 14 to day 42 or typical market age (Schmidt et al. 2009). The days discussed fall into the second growth phase. These days were also chosen as part of a larger heat stress study conducted in our laboratory. The pituitary gland is a complex tissue in that it contains many different cell types, and signals are sent through neural endocrine, paracrine and autocrine networks. The messages received by the pituitary gland lead to many changes within the tissue and results in tissue plasticity. The results of this analysis identify the expression levels of genes in the pituitary gland that are either more abundant or are unique to this particular tissue at specific points in development. These data confer a better understanding of the genes and pathways that are critical to the function of the pituitary gland.

\section{Materials and methods}

\section{Animal housing and tissue collection}

Male Ross 708 broiler chickens (Gallus gallus) were obtained on the day of hatch from Mountaire Hatchery (Millsboro, DE, USA) and placed in large-colony houses on the University of Delaware farm (Newark, DE, USA). Husbandry and management followed all standards and procedures as approved by the Animal Care and Use Committee (AACUC \#(27) 03-12-14R). Standard broiler feed (corn-soy), which met all NRC requirements (National Research Council 1994), and water were supplied, and animals had ad libitum access throughout the trial. Birds were humanely killed and the pituitaries were collected on 21, 22 and 42 days post-hatch as part of a larger study. Whole pituitaries were placed into liquid nitrogen and stored at $-80^{\circ} \mathrm{C}$ until further analysis.

\section{RNA isolation, cDNA synthesis, Rnaseq library preparation and qRT-PCR validation}

Total RNA was extracted from whole pituitary glands (8-20 mg depending on age) from individual birds using the Qiagen RNeasy Mini Kit (Germantown, MD, USA).
Total RNA quantity was measured using a Qubit Fluorometer, and quality was assessed by fragment analysis. A total of 15 pituitary glands from day 21, 10 from day 22 and 10 from day 42 were used for individual Rnaseq library preparation using the Illumina Stranded RNAseq kit (San Diego, CA, USA). All 35 samples were sequenced at the Delaware Biotechnology Institute (DBI) Sequencing and Genotyping center using the Illumina HiSeq 2500 sequencer. All libraries were sequenced to a depth of 20-30million reads per library. The reads were aligned to the Gallus gallus, ver 4 genome sequence, identified and counted using software packages Bowtie, Tophat and Cufflinks, and expression levels were reported as fragments per kilobase of gene per million mapped reads (FPKM) values for further analysis (Trapnell et al. 2009, Langmead \& Salzberg 2012). Three biological replicates for each day (day 21, 22 and 42) were used for cDNA synthesis using the SuperScript First-Strand Synthesis System for RT-PCR (Invitrogen). cDNA concentration was determined using the Qubit Fluorometer and diluted to $30 \mathrm{ng} / \mu \mathrm{L}$ for PCR. qRT-PCR was performed using Fast SYBR green master mix (Applied Biosystems) on the Applied Biosystems 7500 Fast Real-Time PCR system for the following genes: glycoprotein alpha subunit $(C G A)$, follicle-stimulating hormone beta $(F S H B)$, growth hormone $(G H)$, proopiomelanocortin $(P O M C)$, prolactin $(P R L)$ and thyroid-stimulating hormone beta (TSHB). Each qRT-PCR (Table 1) was performed in triplicate, and analysis was completed using the delta Ct method. All sequencing data generated in the current project have been uploaded in the NCBI Gene Expression Omnibus application and are accessible through GEO series, accession number GSE89297.

\section{Relative tissue expression analysis}

The gene expression of the pituitary gland was compared to the expression of other tissues (abdominal fat pad, heart fat pad, breast muscle, cerebellum, heart, liver, duodenum, jejunum, ileum, spleen, retina, pineal and hypothalamus) analyzed by our laboratory following the relative tissue expression ( $\mathrm{rEx}$ ) protocol outlined by Bailey and coworkers (Bailey et al. 2009). Each day the expression was analyzed independently, and maximum FPKM values for each gene in the pituitary gland were compared to the median FPKM value of each gene in all other collected tissues with the following calculation:

$$
\log _{2} \frac{\text { Maximum FPKM for each genein pituitary gland }}{\text { Median FPKM for each gene in all other collected tissues }}
$$


Table 1 Primer sequences used for qRT-PCR transcriptome validation.

\begin{tabular}{ll}
\hline Gene & \multicolumn{2}{l}{ Forward primer } \\
\cline { 2 - 2 } CGA & 5' GCAACGTGCTGTGTAGCAAAG 3' \\
FSHB & 5' CGTACAGGGTAGAGCCAACGA 3' \\
GH & 5' GCTTCAAGAAGGATCTGCACAA 3' \\
$P O M C$ & 5' GCTACGGCGGCTTCATGA 3' \\
$P R L$ & 5' TTGGGCGGGTTCATTCTG 3' \\
$T S H B$ & 5' TGGCCATCAACACCACCAT 3' \\
\hline
\end{tabular}

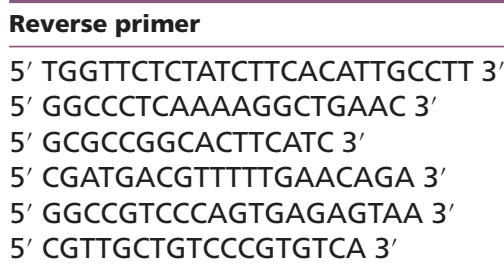

Primer sequences used in qRT-PCR validation of transcriptomic data comparing expression levels between days 21, 22 and 42 . Alpha subunit of glycoproteins $(C G A)$, follicle-stimulating hormone, beta (FSHB), growth hormone $(G H)$, proopiomelanocortin (POMC), prolactin (PRL) and thyroid-stimulating hormone, beta $(T S H B)$.

The $\log _{2}$ distribution was used to normalize the data and apply a $t$-test. Resulting genes that were greater than two standard deviations from the mean and with a $P$ value less than 0.05 were considered enriched in the pituitary gland. The enriched expression of these particular genes is thought to provide the unique functions of the pituitary gland or is critical to the overall function of the pituitary gland at that point in development. The enriched genes were uploaded to AmiGO 2 for gene ontology (GO) term analysis (Carbon et al. 2009), PathRings for pathway analysis (Zhu et al. 2015) and WebGIVI for text mining and the identification of iTerms (L Sun \& CJ Schmidt, unpublished observations).

\section{Results}

Starting gene lists and final enriched genes by day are available in Supplementary Tables 1, 2, 3, 4, 5 and 6 (see section on supplementary data given at the end of this article) (Supplementary Tables 1, 2 and 3; starting gene list for days 21, 22 and 42, respectively; Supplementary Tables 4, 5 and 6; final enriched gene list for days 21, 22 and 42 , respectively). Figure 1 shows a Venn diagram of the number of enriched pituitary genes shared (intersects) and differing (complement) between time points. A total of 25 enriched pituitary genes were shared between all three time points. Day 21 and day 22 were chosen for collection as part of a larger study and share 295 enriched genes compared to all other tissues. By day 42 , there are 407 enriched genes not shared with day 21 and 22 .

\section{Day 21 and day 22: 295 shared genes}

The 295 enriched genes shared between day 21 and day 22 grouped into the following GO terms: structure development (75 genes), neurogenesis (35 genes), neuron differentiation (26genes) and nervous system development (43 genes). More specific GO terms associated with the
295 shared genes are neuron cell-cell adhesion (4 genes), oligodendrocyte differentiation (8 genes) and regulation of gliogenesis (9 genes). Figure 2 shows PathRings output for 295 shared genes between D21 and D22. Significant pathways and genes are shown in Table 2 .

Several genes within the signal transduction pathway and developmental biology pathway were related to axon connection formation and axonal organization (CNTN1, CNTN2, NFASC, LAMA1 and SLIT1), as well as neurite outgrowth (EGF, ERBB4 and NRG3). Additionally, glutamate (GRM3 and GRM7) and GABA (GABRA3) receptors and genes related to synaptic transmission and messaging (ASIC4, HRH3 and CHL1) were enriched at days 21 and 22 when compared to all other tissues.

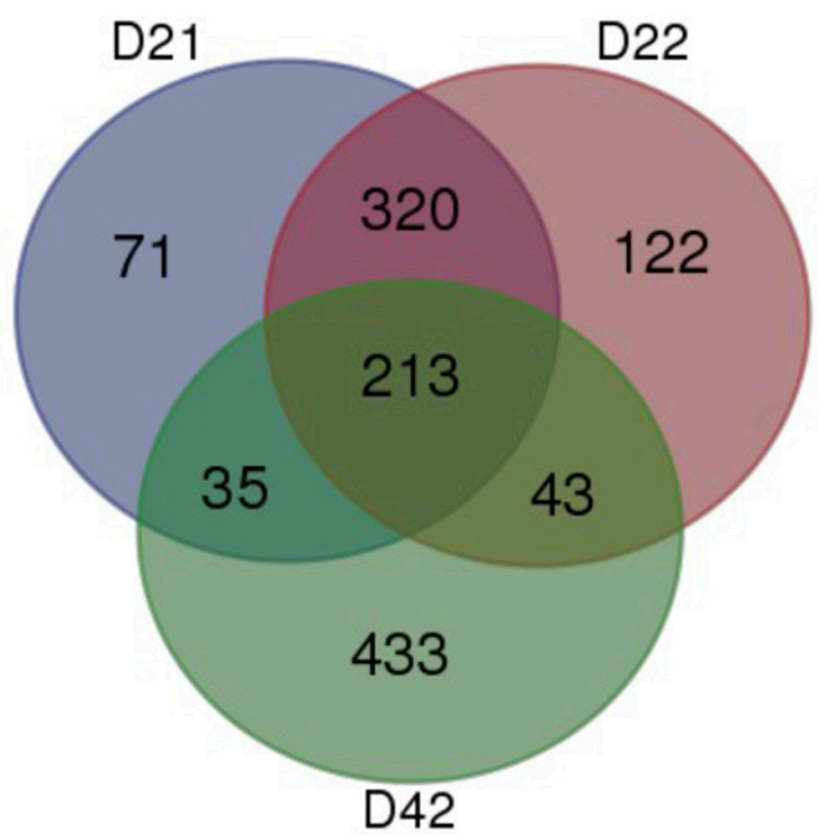

\section{Figure 1}

Distribution of enriched genes in the chicken (Gallus gallus) pituitary gland by day. Total number of enriched genes after relative tissue expression analysis in the whole pituitary gland at post-hatch days 21, 22, and 42.

Published by Bioscientifica Ltd. 


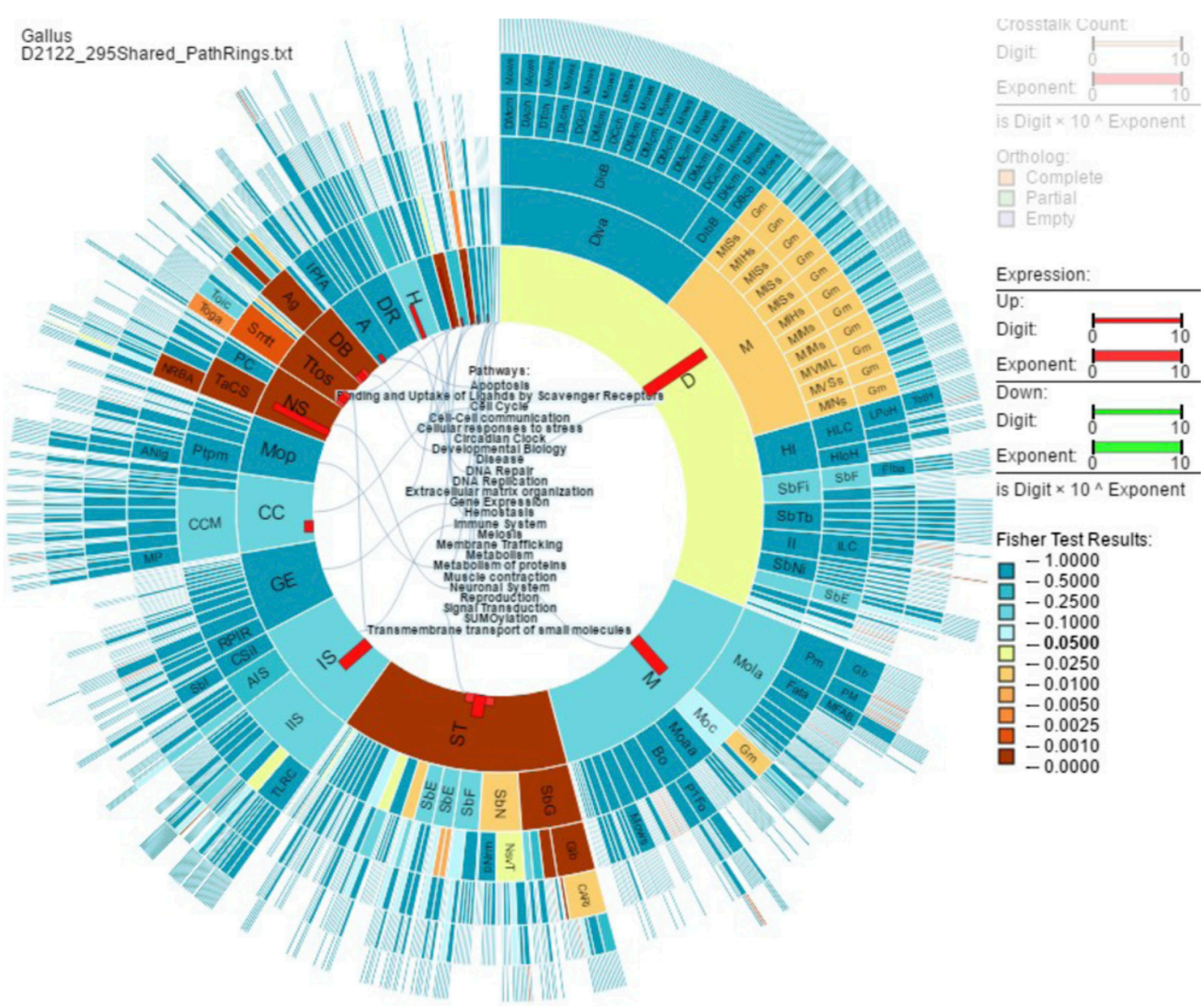

\section{Figure 2}

PathRings output for 295 shared enriched genes between days 21 and 22 in chicken (Gallus gallus) pituitary glands. Significance, determined by the Fisher Exact Test, is indicated by color. Blue is not significant while yellow to maroon is $P$-value $0.05-0.0001$, respectively. DB, developmental biology; NS, neuronal system; ST, significant pathways are signal transduction; Ttos, transmembrane transport of small molecules.

\section{Day 21: 74 genes; day 22: 92 genes}

Days 21 and 22 share a large portion of enriched genes; however, there are genes unique to each day. Further investigation of the individual days in AmiGO2 indicates an enrichment of genes associated with neuronal morphogenesis and synapse structure and organization at day 21 (SYNDIG1, RELN and LRRTM1). At day 22, the uniquely enriched genes are related to neurite outgrowth, axonal migration, oligodendrocyte

Table 2 Significant pathways in PathRings and the enriched genes within each pathway for 295 shared genes between days 21 and 22 in the chicken (Gallus gallus) pituitary gland.

\section{Pathway}

Signal transduction

Neuronal system

Transmembrane transport of small molecules

Developmental biology

\section{Gene symbol}

ADORA1, ARHGEF4, CHRDL1, CNTN1, COL9A3, EGF, ERBB4, FZD10, GFAP, GNAO1, GPR17, GREM2, GRM3, GRM7, GRPR, HRH3, NPBWR1, NRG3, NTRK2, PDE4A, PDE8B, RAMP1, WNT11, WNT6, WNT7B

CACNG4, CHRNA4, GABRA3, GRIA4, GRIK2, GRIK3, KCNK2

ANO4, AQP4, ASIC4, GABRA3, SGK2, SLC13A5, SLC15A2,

SLC26A4, SLC30A8, SLC44A5, SLC6A9

ANK2, CHL1, CNTN1, CNTN2, COL9A3, LAMA1, NFASC, NTN1, SLIT1, UNC5A

DB, developmental biology; NS, neuronal system; ST, signal transduction; Ttos, transmembrane transport of small molecules.

\begin{tabular}{|lr}
\hline http://jme.endocrinology-journals.org & ○ 2017 Society for Endocrinology \\
DOI: $10.1530 / J M E-16-0186$ & Printed in Great Britain
\end{tabular}


Table 3 Gene ontology (GO) terms and enriched genes within each term for chicken (Gallus gallus) day 42 pituitary glands.

\begin{tabular}{|c|c|c|}
\hline Gene ontology (GO) term & \# of genes in GO term & Gene symbol \\
\hline Pituitary gland development & 11 & $\begin{array}{l}\text { DRD2, GHRHR, ISL1, LHX3, PAX6, PITX1, } \\
\text { PITX2, POU1F1, SIX3, TBX19, WNT5A }\end{array}$ \\
\hline Endocrine system development & 16 & $\begin{array}{l}\text { CGA, CRHR1, DRD2, GHRHR, ISL1, LHX3, } \\
\text { NR5A1, PAX6, PITX1, PITX2, POU1F1, } \\
\text { RFX6, RFX8, SIX3, TBX19, WNT5A }\end{array}$ \\
\hline Regulation of hormone levels & 17 & $\begin{array}{l}\text { CGA, CPE, CRHR1, DIO2, DRD2, ESR1, } \\
\text { FOXL2, GHRHR, GHSR, ILDR1, INHBA, } \\
\text { NR5A1, PASK, PCSK1, POMC, PRL, RFX6 }\end{array}$ \\
\hline Cell-cell signaling & 24 & $\begin{array}{l}\text { C2CD4C, CACNA1B, CADPS, CGA, CHRNA3, } \\
\text { CPE, CRHR1, DRD2, GABRG1, GHRHR, } \\
\text { GHSR, GPR149, HTR1B, INHBA, KLF4, } \\
\text { LHX5, LOC100858799, P2RX2, PDYN, } \\
\text { PENK, PITX2, POMC, SYT4, WNT5A }\end{array}$ \\
\hline
\end{tabular}

differentiation and myelination $(F A 2 H, G L D N, P L P 1$, EPHA2, PTGDS, PTGS1 and STMN4). Two immunerelated genes were also enriched in day 22: histone deacetylase 11 (HDAC11) and complement regulatory protein CD59 molecule (CD59). Although these genes were uniquely enriched in the pituitary gland at day 22, the mean FPKM for these genes at days 21 and 22 were both high in comparison to day 42 ((HDAC11: day 21: 75.27, day 22: 84.64, day 42: 6.53); (CD59: day 21: 467.2, day 22: 505.7, day 42: 54.0)).

\section{Day 42: 407 genes}

At day 42, there are 407 unique genes with the following GO terms: pituitary gland development (11 genes), endocrine system development (15 genes), hormone metabolic process and regulation of hormone levels (28 genes) and cell-cell signaling (18 genes) (Table 3 ). In addition to endocrine-related GO terms, neurogenesis (34 genes) and nervous system development (42 genes), GO terms were also present within day 42 enriched genes. Figure 3 shows the pituitary-specific iTerms and genes output from WebGIVI for the day 42 time point. At day 42, expression of endocrine-related genes such as $G H, P R L, C G A$, TSHB, FSHB and POMC increased and the following fold changes were seen in the transcriptomic data between days 22 and 42: GH 2700-fold, PRL 3000-fold, CGA (alpha subunit for FSHB, TSHB LHB) 1600-fold, TSHB 2300-fold, FSHB 6800-fold and POMC 2600-fold. To validate these robust transcriptomic changes, qRT-PCR results are summarized in Fig. 4.

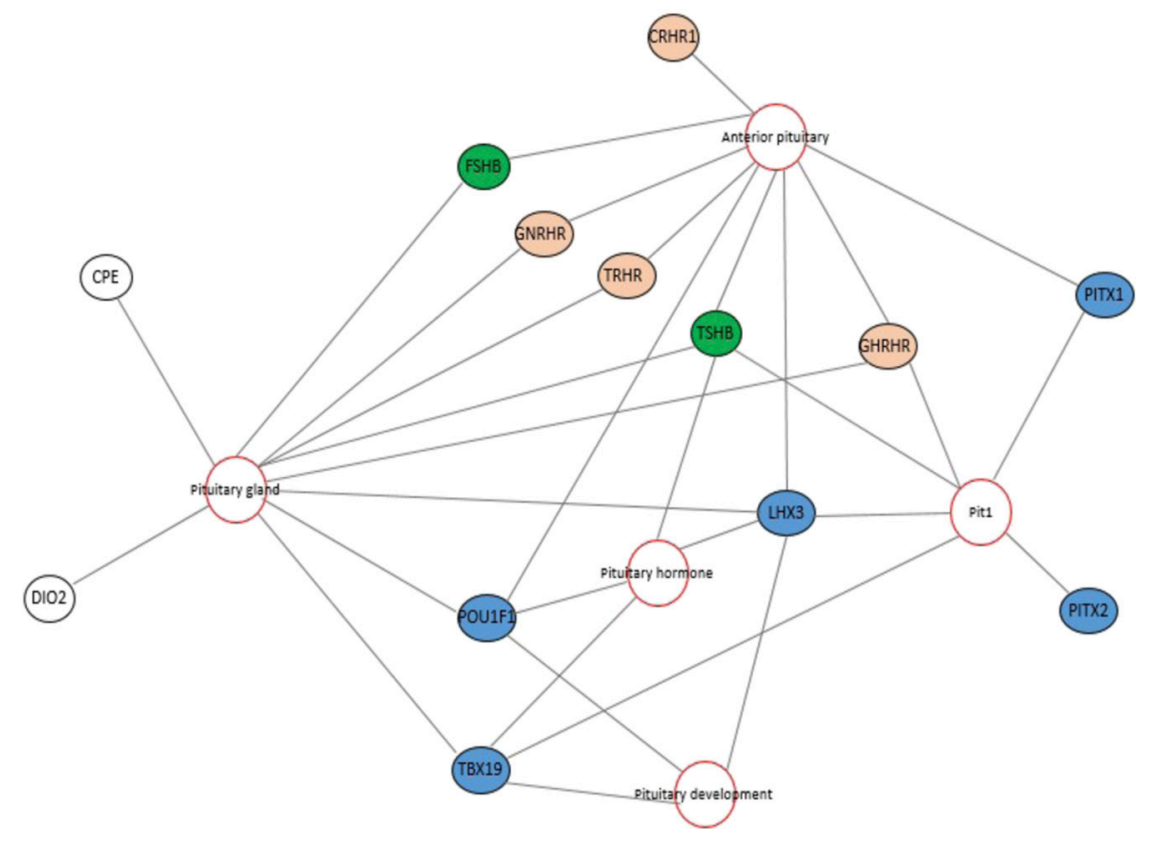

Figure 3

Adapted WebGIVI output for chicken (Gallus gallus) pituitary iTerms and enriched genes expressed at day 42. Adapted WebGIVI output for general 'pituitary' iTerms. Red circles indicate iTerms generated by WebGIVI. Black outlined circles indicate genes from the enriched gene input list. Gray lines (edges) show connections between genes and iTerms. Blue filled genes are pituitary transcription factors. Orange filled genes are receptors for hypothalamus releasing hormones. Green filled genes are beta subunits of follicle stimulating hormone (FSH) and thyroid stimulating hormone (TSH). http://jme.endocrinology-journals.org DOI: 10.1530/JME-16-0186
○ 2017 Society for Endocrinology Printed in Great Britain 


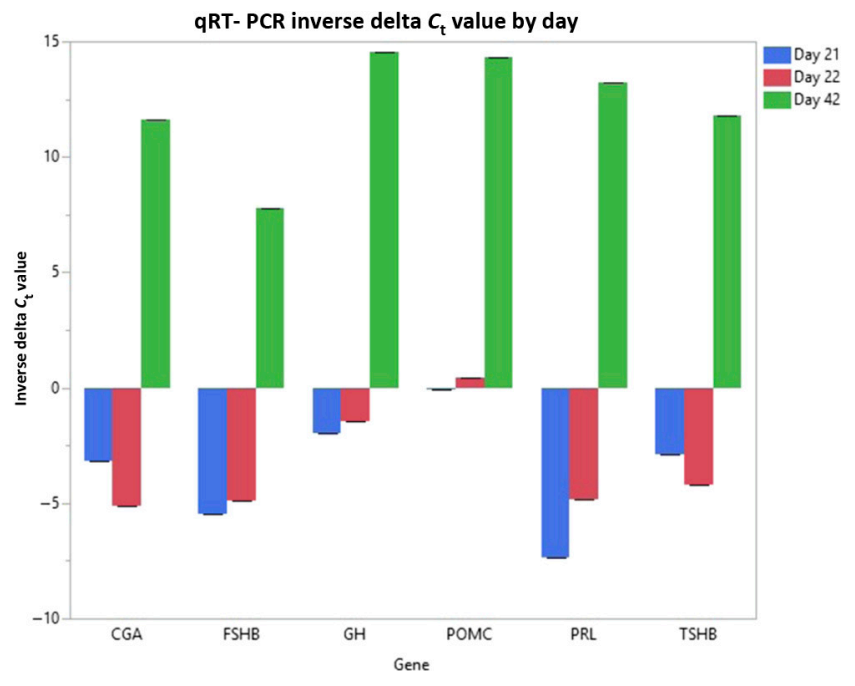

Figure 4

Inverse Delta $C_{\mathrm{t}}$ values for six genes comparing days 21,22 , and 42 chicken (Gallus gallus) post-hatch pituitary gene expression. Alpha subunit of glycoproteins (CGA), follicle stimulating hormone, beta (FSHB), growth hormone $(G H)$, pro-opiomelanocortin (POMC), prolactin (PRL), and thyroid stimulating hormone, beta (TSHB) were used to validate increased expression seen in the transcriptome between days 21, 22, and 42. Inverse Delta $C_{\mathrm{t}}$ values are shown. Errors bars constructed using one standard error from the mean.

\section{Day 21, day 22 and day 42: 25 shared genes}

The 25 enriched genes mean FPKM values for each day are shown in Table 4. Enriched genes were uploaded to WebGIVI (Fig. 5) and AmiGO2 GO terms system development (COL11A1, DNAH5, GPC3, SIX6, SOX3 and TRAPPC4) and sensory organ development (COL11A1, SIX6 and SOX3). These genes include transcription factors and genes whose products code for structural proteins and are associated with the extracellular matrix.

\section{Transcription factors}

Table 5 shows the transcription factors (TF) enriched by day. In the 295 expressed genes shared between days 21 and 22, there are 14 enriched transcription factors. AmiGO 2 analysis resulted in GO terms negative regulation of neurogenesis and negative regulation of nervous system development (NTN1, PHOX2B, SOX8 and VAX1). Thirty transcription factors were enriched at day 42 when compared to all other tissues. AmiGO2 analysis resulted in two notable GO terms: pituitary gland development (LHX3, TBX19, POU1F1, PAX6, ISL1, PITX1, PITX2 and SIX3) and endocrine system development (8 genes listed previously and NR5A1 and RFX6). Two transcription factors were present in all three days studied: Myelin Transcription Factor 1 (MYT1) and NK2
Homeobox 2 (NKX2-2). MYT1 is a DNA-binding protein with an expression pattern that indicates a potential role in regulating oligodendrocyte differentiation. In the developing and adult central nervous system, MYT1 expression correlates with oligodendrocyte cell growth (Nielsen et al. 2004). NKX2-2 is a gene associated with gliogenesis and may play a role in differentiation of oligodendrocyte progenitor cells (Rousseau et al. 2006).

\section{Discussion}

\section{Day 21 and 22: shared and unique enriched genes}

Relative tissue expression analysis for days 21 and 22 results in similarly enriched genes involved in nervous tissue development (axon formation and neurite outgrowth) and signaling (GABA and glutamate receptors). Acid sensing ion channel subunit family member 4 (ASIC4) is a sodium channel involved in signal transduction and is known to have highest expression in the brain (Grunder et al. 2001). Histamine Receptor H3 (HRH3) is a receptor for histamine, which is involved in neurotransmitter release and pituitary hormone secretion (Nuutinen \& Panula 2010). Uniquely enriched genes at days 21 and 22 indicate a shift from neuronal morphogenesis and synapse organization at day 21 to neurite outgrowth and myelination at day 22 . This may be indicative of a developmental change from morphogenesis to maintenance of the nervous tissue, and it is unclear if this fluctuation between morphogenesis and maintenance continues throughout development. Two immune-related genes were also enriched in day 22: HDAC11 and CD59. Histone deacetylases are a group of enzymes that interact with histones to regulate transcription. HDAC11 is the most novel of the group and is placed in its own category, class IV. HDAC11 regulates the expression of interleukin 10 (IL10), and immune tolerance and suppression can promote IL10 expression in mice macrophages, whereas chromatin changes in macrophages increased HDAC11 recruitment to IL10 gene, inhibiting IL10 production (Villagra et al. 2009, Wang et al. 2011). Studies investigating HDAC11 found abundant transcripts are detected in the brain, skeletal muscle, kidney and testis; however, little is known about its function in healthy tissues (Deubzer et al. 2012). Because HDAC11 is a unique histone deacetylase, and it is possible it has functions that are unknown or not typically associated with histone deacetylases (Gao et al. 2002). CD59 is a cell surface glycoprotein that inhibits the assembly of the membrane attack complex (MAC) during

Published by Bioscientifica Ltd 
Table 4 Mean FPKM values for 25 shared genes between days 21, 22 and 42 in the chicken (Gallus gallus) pituitary gland.

\begin{tabular}{l} 
Gene \\
\hline ADIRF \\
CASR \\
COL11A1 \\
DNAH5 \\
FAM135B \\
FAM3B \\
GPC3 \\
LOC101749214 \\
LOC101749680 \\
LOC101749716 \\
LOC101749894 \\
LOC101750727 \\
LOC421690 \\
MATN4 \\
MDGA2 \\
MOV10L1 \\
MYT1 \\
NALCN \\
NKX2-2 \\
RNF182 \\
SIX6 \\
SORCS1 \\
SOX3 \\
TRAPPC4 \\
UMODL1
\end{tabular}

Description
Adipogenesis regulatory factor
Calcium-sensing receptor
Collagen, type XI, alpha 1
Dynein, axonemal, heavy chain 5
Family with sequence similarity 135, member B
Family with sequence similarity 3, member B
Glypican 3
Uncharacterized LOC101749214
Uncharacterized LOC101749680
Uncharacterized LOC101749716
Homeobox protein Nkx-2.2-like
Uncharacterized LOC101750727
Vacuolar protein 8-like
Matrilin 4
MAM domain containing glycosylphosphatidylinositol anchor 2
Mov10l1, Moloney leukemia virus 10-like 1, homolog (mouse)
Myelin transcription factor 1
Sodium leak channel, non-selective
NK2 homeobox 2
Ring finger protein 182
SIX homeobox 6
Sortilin-related VPS10 domain containing receptor 1
SRY (sex determining region Y)-box 3
Trafficking protein particle complex 4
Uromodulin-like 1

\begin{tabular}{c} 
Day 21 \\
\hline 2.16 \\
2.33 \\
17.17 \\
0.55 \\
1.82 \\
5 \\
15.17 \\
1.68 \\
0.53 \\
0.95 \\
17.42 \\
1777.2 \\
0.56 \\
2.2 \\
14.16 \\
4.53 \\
8.5 \\
14 \\
20.34 \\
1.43 \\
53.92 \\
13.6 \\
4.99 \\
36.72 \\
1.3 \\
\hline
\end{tabular}

\begin{tabular}{c}
\hline Day 22 \\
\hline 1.722 \\
2.57 \\
20.74 \\
0.4 \\
1.82 \\
3.98 \\
15.82 \\
1.45 \\
0.76 \\
0.67 \\
20.4 \\
1074.13 \\
0.82 \\
2.1 \\
16.98 \\
3.53 \\
8.9 \\
13.27 \\
25.9 \\
1.53 \\
51.5 \\
14.65 \\
5.04 \\
38.12 \\
1.52 \\
\hline
\end{tabular}

\begin{tabular}{c} 
Day 42 \\
\hline 1.8 \\
2.41 \\
24.49 \\
0.98 \\
2.08 \\
3.4 \\
12.37 \\
0.99 \\
1.21 \\
1.79 \\
16.02 \\
958.83 \\
0.61 \\
7.02 \\
14.25 \\
6.09 \\
7.35 \\
10.73 \\
18.47 \\
3.14 \\
44.8 \\
16.97 \\
3.95 \\
34.17 \\
2.55 \\
\hline
\end{tabular}

Mean FPKM values in the pituitary gland for 25 shared enriched genes in days 21, 22 and 42 when compared to all other tissues (abdominal and heart fat, breast muscle, cerebellum, heart, liver, duodenum, jejunum, ileum, spleen, retina, pineal and hypothalamus).

complement attack (Huang et al. 2001). Activation of the complement attack can lead to inflammation and lysis of cells and needs to be highly regulated. CD59 inhibits MAC osmolytic pore formation thereby protecting cells (Liu et al. 2007). It is possible that development of the pars tuberalis and the macrophages within this region result in enrichment of these immune-related genes at days 21 and 22 but not day 42 and may provide antiinflammatory functions during neuronal development. Overall, the focus of the pituitary gland at days 21 and 22 appears to be focused on developing and maintaining nervous tissue connections and signaling.

\section{Day 42 enriched genes}

By day 42, gene expression suggests a shift in the focus of the pituitary gland through maturation from strictly nervous tissue development and signaling to endocrineand hormone-related functions. Most notably, there is the robust increase in expression of peptide hormone

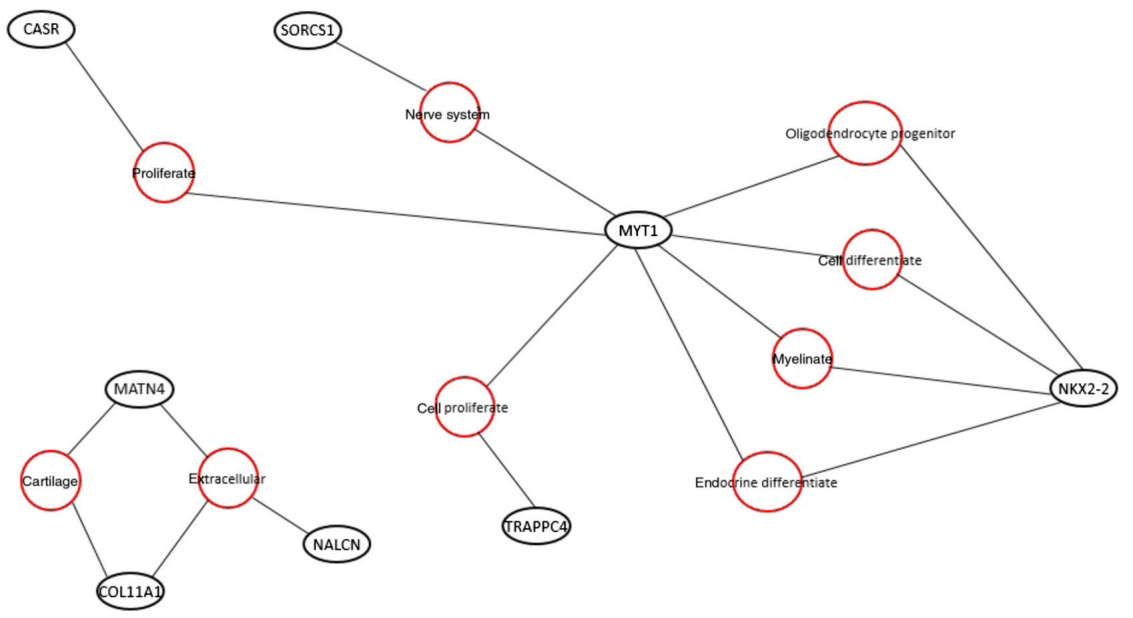

Figure 5

Adapted WebGIVI output for chicken (Gallus gallus) pituitary iTerms and 25 shared enriched genes expressed at days 21, 22 and 42 . Adapted WebGIVI output for 25 genes enriched at all three time points and corresponding iTerms. Red circles indicate iTerms generated by WebGIVI. Black outlined circles indicate genes from the enriched gene input list. Gray lines (edges) show connections between genes and iTerms. 
Table 5 Transcription factors enriched at days 21, 22 and 42 in chicken (Gallus gallus) pituitary glands.

\begin{tabular}{|c|c|c|c|}
\hline Day(s) & \# of enriched genes & TF symbol & TF description \\
\hline \multirow[t]{2}{*}{ Days 21,22 and 42} & 25 genes & NKX2-2 & NK2 homeobox 2 \\
\hline & & MYT1 & Myelin transcription factor 1 \\
\hline \multirow[t]{14}{*}{ Days 21 and 22} & 295 genes & FOXC2 & Forkhead box C2 \\
\hline & & FOXL1 & Forkhead box L1 \\
\hline & & IRX1 & Iroquois homebox 1 \\
\hline & & $\mathrm{LHX} 2$ & LIM homeobox 2 \\
\hline & & MSX1 & Msh homeobox 1 \\
\hline & & NPAS3 & Neuronal PAS domain protein 3 \\
\hline & & NTN1 & Netrin 1 \\
\hline & & OLIG3 & Oligodendrocyte transcription factor 3 \\
\hline & & PHOX2B & Paired like homeobox 2B \\
\hline & & POU3F1 & POU class 3 homeobox 1 \\
\hline & & sox8 & SRY-box 8 \\
\hline & & ST18 & Suppression of tumorigenicity 18 , zinc finger \\
\hline & & TBX22 & T-box 22 \\
\hline & & VAX1 & Ventral anterior homeobox 1 \\
\hline Day 21 & 74 genes & NR2E1 & $\begin{array}{l}\text { Nuclear receptor subfamily } 2 \text { group } E \\
\text { member } 1\end{array}$ \\
\hline \multirow[t]{4}{*}{ Day 22} & 92 genes & DLX1 & Distal-Less homeobox 1 \\
\hline & & HES6 & Hes family BHLH transcription factor 6 \\
\hline & & LOC427656 & Forkhead box protein L1-like \\
\hline & & Sox10 & SRY-box 10 \\
\hline \multirow[t]{30}{*}{ Day 42} & 407 genes & CHURC1 & Churchill domain containing 1 \\
\hline & & DMBX1 & Diencephalon/mesencephalon homeobox 1 \\
\hline & & ESR1 & Estrogen receptor 1 \\
\hline & & FOS & $\begin{array}{l}\text { FBJ murine osteosarcoma viral oncogene } \\
\text { homolog }\end{array}$ \\
\hline & & FOXG1 & Forkhead box G1 \\
\hline & & FOXL2 & Forkhead box L2 \\
\hline & & HES5 & Hes family BHLH transcription factor 5 \\
\hline & & IRF6 & Interferon regulatory factor 6 \\
\hline & & INHBA & Inhibin beta A \\
\hline & & ISL1 & ISL LIM homeobox 1 \\
\hline & & LHX3 & LIM homeobox 3 \\
\hline & & NEUROD1 & Neuronal differentiation 1 \\
\hline & & NEUROG 1 & Neurogenin 1 \\
\hline & & $\mathrm{NHLH} 2$ & Nescient helix-loop-helix 2 \\
\hline & & NR4A3 & $\begin{array}{l}\text { Nuclear receptor subfamily } 4 \text { group A } \\
\text { member } 3\end{array}$ \\
\hline & & NR5A1 & $\begin{array}{l}\text { Nuclear receptor subfamily } 5 \text { group A } \\
\text { member } 1\end{array}$ \\
\hline & & PAX6 & Paired box 6 \\
\hline & & PITX1 & Paired like homeodomain 1 \\
\hline & & PITX2 & Paired like homeodomain 2 \\
\hline & & POU1F1 & POU class 1 homeobox 1 \\
\hline & & PRRX2 & Paired related homeobox 2 \\
\hline & & RAX & Retina and anterior neural fold homeobox \\
\hline & & RBPJL & $\begin{array}{l}\text { Recombination signal binding protein for } \\
\text { immunoglobulin kappa J region like }\end{array}$ \\
\hline & & RFX6 & Regulatory factor $\mathrm{X} 6$ \\
\hline & & RHOXF1 & Rhox homeobox family member 1 \\
\hline & & SIX1 & SIX homeobox 1 \\
\hline & & SIX3 & SIX homeobox 3 \\
\hline & & SMAD7 & SMAD family member 7 \\
\hline & & TBX19 & T-box 19 \\
\hline & & TBX20 & T-box 20 \\
\hline
\end{tabular}

Transcription factors (TF) present within enriched genes separated by day. \# of enriched genes corresponds to total genes (including TF) that were enriched in each category.

http://jme.endocrinology-journals.org DOI: $10.1530 / \mathrm{JME}-16-0186$
() 2017 Society for Endocrinology Printed in Great Britain
Published by Bioscientifica Ltd 
genes GH, PRL, TSHB and FSHB. This change in enriched genes could be in preparation for reproduction. In addition to receptors for releasing hormones from the hypothalamus, and beta subunits of follicle-stimulating hormone $(F S H)$ and thyroid-stimulating hormone (TSH), two other genes of interest are carboxypeptidase $\mathrm{E}(\mathrm{CPE})$ and deiodinase type 2 (DIO2). CPE is a carboxypeptidase B-like enzyme involved in peptide and neurotransmitter synthesis and processing of neuropeptides. Immunohistochemical studies in the rat have shown immunoreactivity within all components of the hypothalamic-neurohypophyseal system (Lynch et al. 1990). CPE has prohormone processing functions in neuroendocrine cells and directs proopiomelanocortin $(P O M C)$ to the secretory pathway where it is later cleaved to produce ACTH (Dhanvantari et al. 2002). DIO2 converts thyroxine $\left(\mathrm{T}_{4}\right)$ to triiodothyronine $\left(\mathrm{T}_{3}\right)$, the main metabolic hormone important for neuronal development and differentiation in the brain (Noda 2015). Circulating levels of $\mathrm{T}_{4}$ are transported across the blood-brain barrier to astrocytes by organic aniontransporting polypeptide (OATPs) transporters and converted by DIO2 to $\mathrm{T}_{3}$. $\mathrm{T}_{3}$ is important for neuron, microglia, astrocyte and oligodendrocyte development and function and thyroid hormone dysfunction can influence these processes (Karlsson et al. 2016). DIO2 expression is also involved in seasonal reproduction in birds and is expressed during long day conditions. The increased day length leads to an increase in $\mathrm{T}_{3}$ which then stimulates the release of FSH and luteinizing hormone (LH) for reproduction (Karlsson et al. 2016). The exact location of $\mathrm{DIO} 2$ expression in the brain is still under investigation although it is thought to be exclusively expressed in the glial compartment (Egri et al. 2016). This research shows expression in the pituitary gland suggesting future work should identify the location of DIO2 mRNA within the pituitary.

Dopamine receptor D2 (DRD2) and somatostatin receptor 5 (SSTR5) are receptors found in the brain. DRD2 is expressed in lactotrophs and inhibits prolactin release. SSTR5 is involved in the regulation of GH and TSH. Both DRD2 and SSTR5 are also involved in ACTH release in pituitary tumors (Tani et al. 2011).

Calcium-dependent secretion activator (CADPS) and synaptotagmin IV (SYT4) gene products are involved in the calcium-dependent vesicle-mediated exocytosis of neurotransmitters and peptides in conjunction with plasma membrane-associated and vesicle integral membrane SNAREs. CADPS is essential in exocytosis of dense-core vesicles (used for exocytosis of biogenic amines and peptides) in the pituitary gland and is involved in secretion of serotonin and other neurotransmitters in Caenorhabditis elegans and Drosophila melanogaster (Wassenberg \& Martin 2002). SYT4 is a synaptotagmin that is a member of the vesicle integral membrane SNARE genes. Once hormones are produced, they are stored in secretory granules where extracellular signals induce fusion with the plasma membrane and subsequent release (Moore et al. 2002). SYT4 is an inhibitor of regulated exocytosis (Wassenberg \& Martin 2002).

Cocaine- and amphetamine-regulated transcript (CARTPT) is an anorectic peptide that is expressed in the hypothalamus and the anterior pituitary. In the anterior pituitary, CARTPT is present in corticotrophs, gonadotrophs, lactotrophs and thyrotrophs. CARTPT mRNA expression is regulated by corticosterone and corticotropin-releasing hormone $(\mathrm{CRH})$ from the hypothalamus via corticotropin-releasing hormone receptor 1 (CRHR1). Intracerebroventricular injections of CARTPT lead to elevated ACTH and corticosterone levels, suggesting that CARTPT can activate the hypothalamic-pituitary-adrenal (HPA) axis and may be involved in the processes of the HPA axis, such as the stress response (Mo et al. 2015). Similarly, gastrin-releasing peptide $(G R P)$ is a neuropeptide that affects AVP and CRH secretion from the hypothalamus and acts directly at the pituitary gland by increasing the effects of AVP and CRH release on corticotrophs (Olsen et al. 1992).

\section{Day 21, 22 and 42: 25 shared enriched genes}

Of the 25 shared genes between days 21, 22 and 42 , genes related to the extracellular matrix (ECM) were enriched. The ECM organizes and fills the space between cells and is composed of glycosaminoglycans and fibrous proteins such as collagen and elastin. The ECM not only provides support to surrounding cells but also influences proliferation, differentiation and survival (Alberts et al. 2002). ECM gene collagen type XI alpha 1 (COL11A1) encodes for an alpha chain of a minor fibrillary collagen and is important for extracellular matrix organization and has been associated with cell proliferation and migration (Mio et al. 2007, Shen et al. 2016). Matrilin-4 (MATN4) is in the matrilin family of proteins and is a recently characterized extracellular matrix protein that is expressed in a variety of tissues, including the brain (Wagener et al. 2001). The pituitary gland is involved in nervous tissue and endocrine

Published by Bioscientifica Ltd. 
signaling and displays tissue plasticity in response to signals from the hypothalamus. This plasticity is necessary to accommodate the conditions of development (i.e. initiation reproductive maturation), and the ECM is an important component of tissue growth and maintenance throughout life. In addition to enrichment for specific ECM genes, there are also enriched genes involved in cell proliferation and differentiation. Glypican 3 (GPC3) interacts with the ECM to influence cell cycle control. GPC3 is a glypican member within the family of heparin sulfate proteoglycans anchored to the cell surface that interact with extracellular ligands to influence cell processes, like growth, and function during morphogenesis and tissue repair (Bernfield et al. 1999). Glypicans are predominately expressed in the central nervous system and have been associated with negative regulation of cell proliferation (Sung et al. 2003).

\section{Enriched transcription factor genes}

Transcription factors (TF) control the rate of transcription and are essential to pituitary cell development, differentiation and function (Mizokami et al. 2008). Because of their influential functions, transcription factors that may be associated with particular points in development of the pituitary gland were identified. Within the 295 enriched genes shared between days 21 and 22, 14 transcription factors were enriched. Netrin 1 laminin related (NTN1) and ventral anterior homeobox 1 (VAX1) are both related to axon guidance in the developing nervous system. NTN1 specifically directs the development and migration of luteinizing hormone-releasing hormone neurons in the forebrain (Schwarting et al. 2004). During embryonic development, VAX1 is responsible for limiting the areas in which fibroblast growth factor 10 (FGF10) can induce pituitary-specific development and ensures that only a single pituitary develops (Bharti et al. 2011). Paired like homeobox 2B (PHOX2B) is critical during embryonic development to regulate neural tube progenitor cells to exit the cell cycle and differentiation to a particular type of neuron (Dubreuil et al. 2000). Two transcription factors are associated with neurogenesis (POU3F1 and NPAS3), whereas suppression of tumorigenicity 18 (ST18) is uniformly expressed in the brain and is associated with pro-apoptotic and pro-inflammatory functions in fibroblasts (Yang et al. 2008). Lastly, two genes are associated with the alpha peptide of glycoprotein hormones (CGA). In humans and mice,
Msh Homeobox 1 (MSX1) has been associated with CGA expression and is localized in growth hormone and thyroid-stimulating hormone cells of the anterior pituitary (Mizokami et al. 2008). LIM Homeobox 2 (LHX2) is a transcription factor known to repress the CGA promoter (Susa et al. 2006) and has a direct role in the development of the posterior pituitary gland (Davis et al. 2010). Several transcription factors normally associated with embryonic development at days 21 and 22 were identified; however, the presence of these genes in post-hatch birds suggests these genes may continue to influence development through axonal guidance (VAX1) and limiting the growth of pituitary-related cell types (NTN1).

Thirty transcription factors are enriched at day 42 and several are involved in endocrine-related processes. POU class 1 homeobox 1 (POU1F1) is a pituitary-specific transcription factor that is responsible for the activation of GH, PRL and TSHB gene transcription (Pfaffle et al. 1993) and is critical for proper pituitary development (Zhu et al. 2005). Nuclear receptor subfamily 5 group A member 1 (NR5A1) functions in the development of LH- and FSH-expressing cells and T-box 19 (TBX19) is restricted to corticotrophs where it binds the POMC promoter and affects the expression of ACTH (Zhu et al. 2005). Lim homeobox 3 (LHX3) and Forkhead box L2 (FOXL2) are associated with positive regulation of the CGA promoter (Susa et al. 2006). Neuron differentiation 1 (NEUROD1) has been identified as a transcription factor involved in corticotroph differentiation and POMC gene expression (Tani et al. 2011). Regulatory factor X6 (RFX6) is a newly characterized transcription factor expressed primarily in pancreatic tissues and may regulate pancreatic development. The paralogous gene, regulatory factor $\mathrm{X} 7$ ( $R F X 7)$, is also recently characterized in humans and is expressed in several tissues, particularly the brain (Aftab et al. 2008) but was not shown to be enriched in our data. It is surprising that a transcription factor most commonly associated with the pancreas $(R F X 6)$ is present in the pituitary gland at day 42 rather than the paralogous transcription factor normally associated with brain tissue (RFX7); however, the brain is an insulin-sensitive organ and perhaps $R F X 6$ is present in the chicken pituitary gland to assist in insulin-related processes (i.e. insulin receptors, glucose uptake).

Two pituitary enriched transcription factors found in all days, MYT1 and NXK2-2, are both related to oligodendrocyte differentiation, proliferation and maturation. MYT1 is a DNA-binding protein with

Published by Bioscientifica Ltd 
an expression pattern that indicates a potential role in regulating oligodendrocyte differentiation. In the developing and adult central nervous system, MYT1 expression correlates with oligodendrocyte cell growth (Nielsen et al. 2004). NKX2-2 is associated with gliogenesis and may play a role in the differentiation of oligodendrocyte progenitor cells (Rousseau et al. 2006). The presence of these TFs in all days indicates the need for continued oligodendrocyte proliferation. This proliferation may accommodate new connections between the pituitary gland and other brain regions, specifically the hypothalamus, and may be important for establishing and maintaining the connections within the hypothalamo-pituitary axis. Other neuronalrelated TFs independent of those identified at days 21 and 22 were enriched at day 42 (i.e. CHURC1, DMBX1). These transcription factors should be explored further to determine the temporal relationship as it is possible these TFs are necessary to establish the endocrine-related processes within the pituitary gland. TF results also point to a maturation shift from prominently neuronal-related TFs at day 21/22 to endocrine-related TFs at day 42 .

In summary, there is a transcriptome focus on nervous tissue development, signaling and maturation at days 21 and 22 post-hatch. By day 42, there is a shift from strictly nervous tissue gene expression toward endocrinerelated processes, possibly indicating maturation in preparation for reproduction. The results of this study lead to valuable insight into the genes involved in the pituitary gland at several points in development, particularly related to maturation. Future work aims to utilize fluorescent in situ hybridization techniques to investigate the location of particular mRNA transcripts within the pituitary such as DIO2 and RFX6. Future work should investigate the transcriptomic differences, particularly related to neuronal and endocrine maturation, as a function of age in male broiler chicken pituitary glands by collecting additional time points in future trials. The pituitary gland is a tissue that contains many different cell types and exerts many physiological changes based on the input received from the body and the environment. Whole tissue transcriptomics is informative but cellular transcriptomic changes that may have important physiological impacts may be lost or not as robust. Single-cell transcriptomic data could further elucidate the cellular transcriptomic differences that may influence the development and function of the pituitary gland. Single-cell transcriptome analysis would allow for identification of more prevalent cell types and the transcriptomic changes throughout development.
Supplementary data

This is linked to the online version of the paper at http://dx.doi.org/10.1530/ JME-16-0186

Declaration of interest

The authors declare that there is no conflict of interest that could be perceived as prejudicing the impartiality of the research reported.

\section{Funding}

This project was supported by Agriculture and Food Research Initiative Competitive Grant 2011-67003-30228 from the United States Department of Agriculture National Institute of Food and Agriculture.

\section{Author contribution statement}

E M P, C J S and S J L conceived and designed the experiments. E M P and C J S performed the experiments. E M P and C J S analyzed the data. E M P, C J S and S J L contributed reagents/materials/analysis tools. E M P wrote the paper.

\section{Acknowledgements}

The authors would like to thank Matheus Reis for his help in conducting the bird trial, members of the Schmidt Laboratory for assistance during necropsies and Dr Erin Brannick and Dr Robert Dyer for aiding in proper collection of the pituitary gland. Special thanks to Schmidt Lab member Blair Schneider for assistance with pituitary gland RNA preparation.

\section{References}

Aftab S, Semenec L, Shih-Chieh Chu J \& Cheng N 2008 Identification and characterization of novel human tissue-specific RFX transcription factors. BMC Evolutionary Biology $\mathbf{8} 226$ (doi:10.1186/1471-2148-8-226)

Alberts B, Johnson A, Lewis J, Raff M, Roberts K \& Walter P 2002 The extracellular matrix of animals. In Molecular Biology of the Cell, edn 4. New York, NY, USA: Garland Science.

Bailey MJ, Coon SL, Carter DA, Humphries A, Kim J, Shi Q, Gaildrat P, Morin F, Ganguly S, Hogenesch JB, et al. 2009 Night/Day changes in pineal expression of $>600$ genes. Central role of Adrenergic/cAMP signaling. Journal of Biological Chemistry 284 7606-7622. (doi:10.1074/jbc.m808394200)

Bernfield M, Gotte M, Park PW, Reizes O, Fitzgerald ML, Lincecum J \& Zako M 1999 Functions of cell surface heparan sulfate proteoglycans. Biochemistry 68 729-777. (doi:10.1146/annurev. biochem.68.1.729)

Bharti K, Gasper M, Bertuzzi S \& Arnheiter H 2011 Lack of the ventral anterior homeodomain transcription factor VAX1 leads to induction of a secondary pituitary. Development 138 873-878. (doi:10.1242/ dev.056465)

Carbon S, Ireland A, Mungall CJ, Shu SQ, Marshall B \& Lewis S 2009 AmiGO: online access to ontology and annotation data. Bioinformatics 25 288-289. (doi:10.1093/bioinformatics/btn615)

Davis SW, Castinetti F, Carvalho LR, Ellsworth BS, Potok MA, Lyons RH, Brinkmeier ML, Raetzman LT, Carninci P, Mortensen AH, et al. 2010 Molecular mechanisms of pituitary organogenesis: In search of novel regulatory genes. Molecular and Cellular Endocrinology 323 4-19. (doi:10.1016/j.mce.2009.12.012)

Published by Bioscientifica Ltd. 
Deubzer HE, Schier MC, Oehme I, Lodrini M, Haendler B, Sommer A \& Witt O 2012 HDAC11 is a novel drug target in carcinomas. International Journal of Cancer 132 2200-2208. (doi:10.1002/ ijc.27876)

Dhanvantari S, Arnaoutova I, Snell CR, Steinbach PJ, Hammond K, Caputo GA, London E \& Loh YP 2002 Carboxypeptidase E, a prohormone sorting receptor, is anchored to secretory granules via a c-terminal transmembrane insertion. Biochemistry 41 52-60. (doi:10.1021/bi015698n)

Dubreuil V, Hirsch MR, Pattyn A, Brunet JF \& Goridis C 2000 The Phox $2 b$ transcription factor coordinately regulates neuronal cell cycle exit and identity. Development 127 5191-5201.

Egri P, Fekete C, Denes A, Reglodi D, Hashimoto H, Fulop BD \& Gereben B 2016 Pituitary adenylate cyclase-activating polypeptide (PACAP) regulates the hypothalamo-pituitary-thyroid (HPT) axis via type 2 deiodinase in male mice. Endocrinology 157 1-11. (doi:10.1210/en.2015-1982)

Gao L, Cueto MA, Asselbergs F \& Atadja P 2002 Cloning and functional characterization of HDAC11, a novel member of the human histone deacetylase family. Journal of Biological Chemistry 277 25748-25755. (doi:10.1074/jbc.M111871200)

Grunder S, Geisler HS, Rainier S \& Fink JK 2001 Acid-sensing ion channel (ASIC) 4 gene: Physical mapping, genomic organisation, and evaluation as a candidate for paroxysmal dystonia. European Journal of Human Genetics 9 672-676. (doi:10.1038/sj. ejhg.5200699)

Huang J, Gou D, Zhen C, Jiang D, Mao X, Li W, Chen S \& Cai C 2001 Protection of xenogeneic cells from human complement-mediated lysis by the expression of human DAF, CD59, and MCP. FEMS Immunology and Medical Microbiology 31 203-209. (doi:10.1111/j.1574-695X.2001.tb00521.x)

Karlsson AC, Fallahshahroudi A, Johnsen H, Hagenblad J, Wright D, Andersson L \& Jensen P 2016 A domestication related mutation in the thyroid stimulating hormone receptor gene (TSHR) modulates photoperiodic response and reproduction in chickens. General and Comparative Endocrinology 228 69-78. (doi:10.1016/j. ygcen.2016.02.010)

Langmead B \& Salzberg SL 2012 Fast gapped-read alignment with Bowtie 2. Nature Methods 9 357-359. (doi:10.1038/nmeth.1923)

Liu G, Zhang J \& Chen X 2007 Molecular and functional characterization of a CD59 analogue from large yellow croaker Pseudosciana crocea. Molecular Immunology 44 3661-3671. (doi:10.1016/j.molimm.2007.04.006)

Lynch DR, Braas KM, Hutton JC \& Snyder SH 1990 Carboxypeptidase E (CPE): immunocytochemical localization in the rat central nervous system and pituitary gland. Journal of Neuroscience 10 1592-1599.

Mio F, Chiba K, Hirose Y, Kawaguchi Y, Mikami Y, Oya T, Mori M, Kamata M, Matsumoto M, Ozaki K, et al. 2007 A functional polymorphism in COL11A1, which encodes the alpha1 chain of type $\mathrm{XI}$ collage, is associated with susceptibility to lumbar disc herniation. American Journal of Human Genetics 81 1271-1277. (doi:10.1086/522377)

Mizokami Y, Egashira N, Takekoshi S, Itoh J, Itoh Y, Osamura RY \& Matsumae M 2008 Expression of MSX1 in human normal pituitaries and pituitary adenomas. Endocrine Pathology 19 54-61. (doi:10.1007/ s12022-008-9021-7)

Mo C, Cai G, Huang L, Deng Q, Lin D, Cui L, Wang Y \& Li J 2015 Corticotropin-releasing hormone $(\mathrm{CRH})$ stimulates cocaine- and amphetamine-regulated transcript gene (CART1) expression through CRH type 1 receptor in chicken anterior pituitary. Molecular and Cellular Endocrinology 5 166-177. (doi:10.1016/j. mce.2015.09.007)

Moore HPH, Andresen JM, Eaton BA, Grabe M, Haugwitz M, Qu MM \& Machen TE 2002 Biosynthesis and secretion of pituitary hormones: dynamics and regulation. Archives of Physiology and Biochemistry 110 16-25. (doi:10.1076/apab.110.1.16.903)

National Research Council 1994 Nutrient Requirements of Poultry, 9th ed. Washington, USA: National Academy Press.

Nielsen JA, Berndt JA, Hudson LD \& Armstrong RC 2004 Myelin transcription factor 1 (Myt) modulates the proliferation and differentiation of oligodendrocyte lineage cells. Molecular and Cellular Neuroscience 25 111-123. (doi:10.1016/j.mcn.2003.10.001)

Noda M 2015 Possible role of glial cells in the relationship between thyroid dysfunction and mental disorders. Frontiers in Cellular Neuroscience 9 1-7. (doi:10.3389/fncel.2015.00194)

Nuutinen S \& Panula P 2010 Histamine in neurotransmission and brain diseases. Advances in Experimental Medicine and Biology 709 95-107. (doi:10.1007/978-1-4419-8056-4_10)

Olsen L, Knigge U \& Warberg J 1992 Gastrin-releasing peptide stimulation of corticotropin secretion in male rats. Endocrinology 130 2710-2716. (doi:10.1210/en.130.5.2710)

Pfaffle RW, Parks JS, Brown MR \& Heimann G 1993 Pit-1 and pituitary function. Journal of Pediatric Endocrinology 6 229-233. (doi:10.1515/ JPEM.1993.6.3-4.229)

Rousseau A, Nutt CL, Betensky RA, Iafrate AJ, Han M, Ligon KL, Rowitch DH \& Louis DN 2006 Expression of oligodendroglial and astrocytic lineage markers in diffuse gliomas: use of YKL-40, APOE, ASCL1, and NKX2-2. Journal of Neuropathology and Experimental Neurology 65 1149-1156. (doi:10.1097/01. jnen.0000248543.90304.2b)

Schmidt CJ, Persia ME, Feierstein E, Kingham B \& Saylor WW 2009 Comparison of a modern broiler line and a heritage line unselected since the 1950s. Poultry Science 88 2610-2619. (doi:10.3382/ps.200900055)

Schwarting GA, Raitcheva D, Bless EP, ACkerman SL \& Tobet S 2004 Netrin 1-mediated chemoattraction regulates the migratory pathway of LHRH neurons. European Journal of Neuroscience 19 11-20. (doi:10.1111/j.1460-9568.2004.03094.x)

Shen L, Yang M, Lin Q, Zhang Z, Zhu B \& Miao C 2016 COL11A1 is overexpressed in recurrent non-small cell lung cancer and promotes cell proliferation, migration, invasion and drug resistance. Oncology Reports 36 877-885. (doi: 10.3892/or.2016.4869)

Sung YK, Hwang SY, Farooq M, Kim JC \& Kim MK 2003 Growth promotion of HepG2 hepatoma cells by antisense-mediated knockdown of glypican-3 is independent of insulin-like growth factor 2 signaling. Experimental and Molecular Medicine 35 257-262. (doi:10.1038/emm.2003.34)

Susa T, Sato T, Ono T, Kato T \& Kato Y 2006 Cofactor CLIM2 promotes the repressive action of LIM homeodomain transcription factor LHX2 in the expression of porcine pituitary glycoprotein hormone alpha subunit gene. BBA Gene Structure and Expression 1759 403-409. (doi:10.1016/j.bbaexp.2006.08.004)

Tani Y, Sugiyama T, Izumiyama H, Yoshimoto T, Yamada T \& Hirata Y 2011 Differential gene expression profiles of POMC-related enzymes, transcription factors and receptors between non-pituitary and pituitary ACTH-secreting tumors. Endocrine Journal 58 297-303. (doi:10.1507/endocrj.K10E-389)

Trapnell C, Pachter L \& Salzberg SL 2009 TopHat:discovering splice junctions with RNA-seq. Bioinformatics 25 1105-1111. (doi:10.1093/ bioinformatics/btp120)

Villagra A, Cheng F, Wang HW, Suarez I, Glozak M, Maurin M, Nguyen D, Wright KL, Atadja PW, Bhalla K, et al. 2009 The histone deacetylase HDAC11 regulates the expression of interleukin 10 and immune tolerance. Nature Immunology 10 92-100. (doi:10.1038/ni.1673)

Wagener R, Kobbe B, Aszodi A, Aeschlimann D \& Paulsson M 2001 Characterization of the mouse matrilin- 4 gene: A $5^{\prime}$ antiparallel overlap with the gene encoding the transcription factor RBP-I. Genomics 76 89-98. (doi:10.1006/geno.2001.6589) http://jme.endocrinology-journals.org

DOI: 10.1530/JME-16-0186
() 2017 Society for Endocrinology Printed in Great Britain
Published by Bioscientifica Ltd 
Wang H, Cheng F, Woan K, Sahakian E, Merino O, Rock-Klotz J, VicenteSuarez I, Pinilla-Ibarz J, Wright JL, Seto E, et al. 2011 Histone deacetylase inhibitor LAQ824 augments inflammatory responses in macrophages through transcriptional regulation of IL-10. Journal of Immunology 186 3986-3996. (doi:10.4049/jimmunol.1001101)

Wassenberg JJ \& Martin TFJ 2002 Role of CAPS in dense-core vesicle exocytosis. Annals of the New York Academy of Science 971 201-209. (doi:10.1111/j.1749-6632.2002.tb04464.x)

Yang J, Siqueira MF, Behl Y, Alikhani M \& Graves DT 2008 The transcription factor ST18 regulates proapoptotic and proinflammatory gene expression in fibroblasts. FASEB Journal 22 3956-3967. (doi:10.1096/fj.08-111013)

Zhu X, Lin CR, Prefontaine GG, Tollkuhn J \& Rosenfeld MG 2005 Genetic control of pituitary development and hypopituitarism. Current Opinion in Genetics and Development 15 332-340. (doi:10.1016/j.gde.2005.04.011)

Zhu Y, Sun L, Garbarino A, Schmidt C, Fang J \& Chen J 2015 PathRings: a web-based tool for exploration of ortholog and expression data in biological pathways. BMC Bioinformatics 16 1-7. (doi:10.1186/ s12859-014-0430-y)

Received in final form 8 November 2016

Accepted 17 November 2016

Accepted Preprint published online 17 November 2016
Published by Bioscientifica Ltd. 